\title{
Mutation of the AAUAAA polyadenylation signal depresses in vitro splicing of proximal but not distal introns
}

\author{
Maho Niwa and Susan M. Berget \\ Marrs McClean Department of Biochemistry, Baylor College of Medicine, Houston, Texas 77030 USA
}

\begin{abstract}
To investigate the relationship between splicing and polyadenylation during the production of vertebrate mRNAs, we examined the effect of mutation of a poly(A) site on splicing of upstream introns. Mutation of the AAUAAA polyadenylation consensus sequence inhibited in vitro splicing of an upstream intron. The magnitude of the depression depended on the magnesium concentration. Dependence of splicing on polyadenylation signals suggests the existence of interaction between polyadenylation and splicing factors. In multi-intron precursor RNAs containing duplicated splice sites, mutation of the poly(A) site inhibited removal of the last intron, but not the removal of introns farther upstream. Inhibition of removal of only the last intron suggests segmental recognition of multi-exon precursor RNAs and is consistent with previous suggestions that signals at both ends of an exon are required for effective splicing of an upstream intron.
\end{abstract}

[Key Words: Polyadenylation signal; splicing; vertebrate RNAs]

Received July 31, 1991; revised version accepted September 10, 1991.

The majority of vertebrate mRNAs undergo both splicing and polyadenylation during maturation. The relationship between the two processing steps has been unclear. Polyadenylation has been reported to occur shortly after RNA polymerase passes the poly(A) cleavage site and prior to splicing (Ford and Hsu 1978; Nevins and Darnell 1978; Weber et al. 1980). Other experiments, however, demonstrate splicing of introns distal to the poly(A) site, both before polyadenylation and during transcription (Berget and Sharp 1978; Keohavong et al. 1982; Green et al. 1983; Mariman et al. 1983; Beyer and Osheim 1988; LeMaire and Thummel 1990), indicating no obligate ordering of the two processing steps. The possibility remained, however, that splicing of the last intron might be coupled to polyadenylation.

Polyadenylation is readily assayed in vitro with precursor RNAs that lack splicing signals. Likewise, splicing is readily assayed with precursor RNAs that lack poly(A) sites. Furthermore, the two activities do not cofractionate (Christofori and Keller 1988; Gilmartin et al. 1988; Kramer 1988; Takagaki et al. 1988), indicating that the two processing steps are catalyzed by different entities. At face value, these results suggest that splicing and polyadenylation are uncoupled. Several recent reports, however, indicate that mutation or deletion of splicing consensus sequences depresses polyadenylation in vivo (Villereal and White 1983; Huang and Gorman 1990).
Using chimeric RNAs competent for both in vitro splicing and polyadenylation, we recently reported that in vitro polyadenylation is stimulated when an intron is placed upstream of a poly(A) site (Niwa et al. 1990). Stimulation required the presence of splicing signals at the $3^{\prime}$ end of the intron but not at the $5^{\prime}$ end. This observation suggests that the presence of the poly(A) site within a terminal exon (and thereby placement downstream of sequences at the $3^{\prime}$ end of the intron) is important for stimulation. It suggests further that the splicing and polyadenylation machineries interact.

A number of mutants with altered polyadenylation signals have been isolated and characterized. Mutation of the conserved AAUAAA element drastically reduces levels of cytoplasmic RNA. Few, if any, studies have investigated the effect of such mutations on the splicing of upstream introns. Here, we report the effect of the presence of a poly $(\mathrm{A})$ site on in vitro splicing.

We found that a wild-type poly(A) site was required for maximal removal of the upstream intron. Mutation of the essential AAUAAA polyadenylation element impaired splicing. Using constructs containing multiple, identical introns we observed that the effect of the poly $(\mathrm{A})$ site was limited to splicing of the last intron; upstream introns were unaffected. We conclude that splicing of $3^{\prime}$-terminal introns is influenced by the presence of a $\operatorname{poly}(\mathrm{A})$ site. 


\section{Results \\ Polyadenylation signals maximize splicing of an upstream intron}

We have been investigating the relationship between splicing and polyadenylation by examining the in vitro processing of simple chimeric precursor RNAs containing both splicing and polyadenylation signals (Niwa et al. 1990; Niwa and Berget 1991). We find that these precursor RNAs are readily recognized in in vitro processing extracts and are both polyadenylated and spliced to produce a product RNA in which both reactions have occurred (referred to here as $\mathrm{S}+\mathrm{A}+\mathrm{RNA}$ ). Either polyadenylation or splicing can occur first, although only the former pathway produces mature doubly processed RNA. RNA that splices first behaves as a dead-end intermediate that cannot be processed further. Choice of the pathway of processing is dependent on the magnesium concentration: Polyadenylation is favored at low magnesium, and the primary product observed is doubly processed RNA. Splicing dominates at higher magnesium, producing considerable RNA product that has undergone only splicing.

To investigate the effect of polyadenylation signals on splicing we compared the splicing activity of a wild-type single-intron precursor RNA (MXSVL) to that of a mutant precursor RNA containing altered polyadenylation signals. The mutant MXSVL contained a single point mutation converting the required polyadenylation consensus sequence AAUAAA to AAGAAA (Fig. 1A). This mutation effectively abolishes polyadenylation of SV40 late-precursor RNA in vivo and in vitro /Sheets et al. 1990|. Figure 1B compares the splicing activities of these chimeric substrates, as well as the splicing activity of a substrate containing only splicing signals (SB2) under the low magnesium conditions that both favor polyadenylation and maximize production of doubly processed RNA from MXSVL (Niwa and Berget 1991). The SB2 splicing template was spliced very poorly under these conditions. In contrast, MXSVL was both polyadenylated and spliced to produce doubly processed product RNA $(A+S+R N A$ in Fig. IB). MXSVL containing the AAGAAA point mutation was not polyadenylated because of the mutation and was only marginally spliced. Thus, the presence of a poly(A) site in the chimeric RNA permitted splicing in conditions under which it would normally not occur, and this splicing was dependent on a wild-type polyadenylation sequence.

To quantitate splicing activity, the gel in Figure 1B was scanned in a Betagen blot analyzer. Determined radioactivity in each band was converted to femtomoles product and plotted versus reaction time (Fig. 1C). Total splicing activity for the wild-type RNA is the sum of the splicing reactions to produce the $S+$ RNA species (RNA that was spliced, but not polyadenylated) and the $\mathrm{A}+\mathrm{S}+$ RNA species (RNA that was spliced and polyadenylated) indicated in Figure 1B. Mutant RNA and RNA containing only splicing signals spliced at a lower rate than did RNA containing wild-type polyadenylation signals, such that less-spliced RNA was observed at all times of reaction.
Wild-type chimeric RNA spliced 5- to 10-fold better than either the mutant RNA or RNA containing only splicing signals. Thus, the presence of a poly(A) site in the chimeric RNA significantly enhanced splicing of the upstream intron. This observation strongly suggests a synergistic relationship between splicing and polyadenylation.

Precursor RNAs have a finite lifetime in in vitro processing extracts. Analysis of the total amount of RNA in the reactions of the three types of precursor indicated that these RNAs all had a similar half-life in extract (data not shown). Therefore, the difference in activity between wild-type and mutant substrates was not the result of differential stability of substrate or product RNA.

Splicing of chimeric RNA was also examined under conditions more standard for measurement of in vitro splicing (Fig. 2). At $1.5 \mathrm{~mm}$ magnesium, greater splicing activity was observed for both MXSVL WT and MXSVL AAGAAA substrates than at lower magnesium (Fig. 2A). For wild-type precursor RNA, half of the precursor was processed by initial polyadenylation and half was processed by initial splicing. Quantitation of splicing activity indicated that total splicing of the wild-type precursor RNA was about twofold higher than splicing of the mutant precursor RNA (Fig. 2B). Thus, even at magnesium concentration favoring splicing, the presence of a wild-type poly(A) site resulted in more splicing than in a similar construct containing a mutated poly $(\mathrm{A})$ site.

Wild-type chimeric RNA produced two types of spliced RNA: RNA that had been spliced but not polyadenylated (S+RNA), and RNA that had been spliced and polyadenylated ( $S+A$ RNA). Figure $2 C$ compares the rates of production of each of these RNA species to the rate of production of spliced RNA by using the AAGAAA mutant precursor RNA. The production of mutant spliced RNA had kinetics of appearance identical to the spliced but not polyadenylated $(S+)$ population from wild-type RNA. The difference in total splicing between mutant and wild-type precursor RNAs was therefore reflected in the presence of a pool of doubly processed $\mathrm{S}+\mathrm{A}+\mathrm{RNA}$ by using wild-type precursor RNA that was not produced from mutant precursor RNA because of the mutated polyadenylation signal. Production of equivalent amounts of S + RNA by mutant and wild-type chimeric RNA, coupled with the inability of the S+ population to polyadenylate (Niwa and Berget 1991), raises the possibility that wild-type RNA was processed by two distinct pathways at moderate magnesium concentrations, one that was dependent on polyadenylation factors and one that was not. This result suggests that observation of optimal coupling of splicing and polyadenylation in vitro requires use of the low magnesium conditions that favor polyadenylation.

If the latter interpretation were true, one might predict that reactions carried out at higher magnesium concentrations under which polyadenylation is disfavored would exhibit no difference between the splicing of wildtype and mutant RNA. To test this hypothesis, processing of MXSVL WT and MXSVL AAGAAA was carried out at $4 \mathrm{~mm}$ magnesium. Little polyadenylation was ob- 
Niwa and Berget

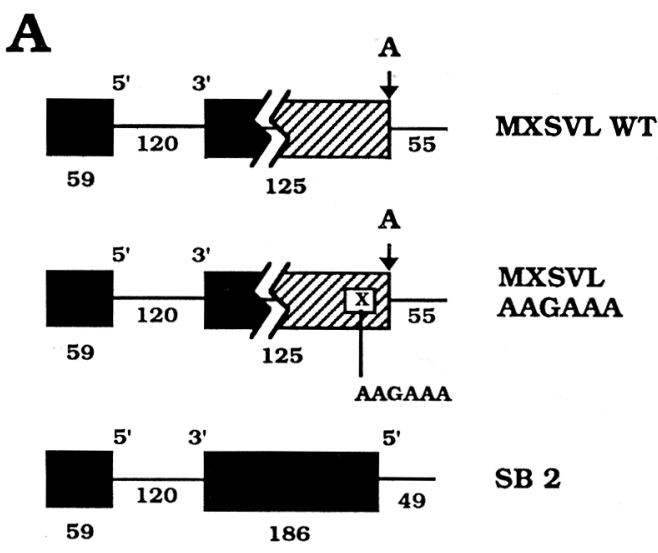

B

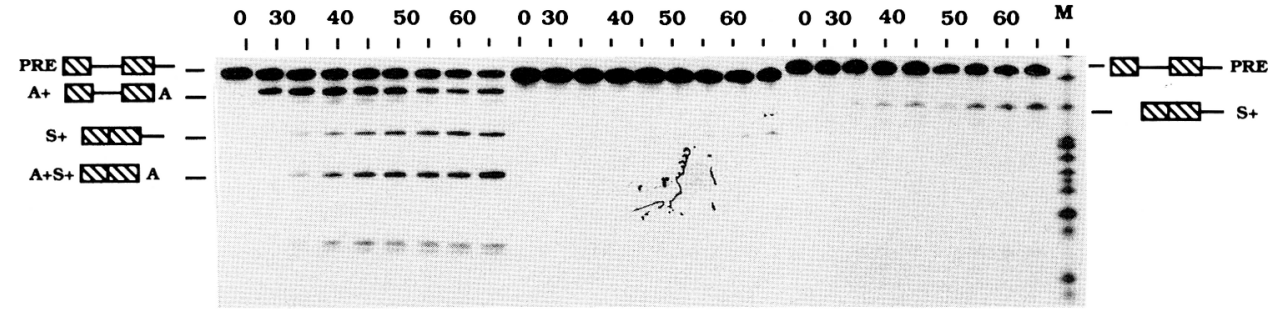

MXSVL WT

C

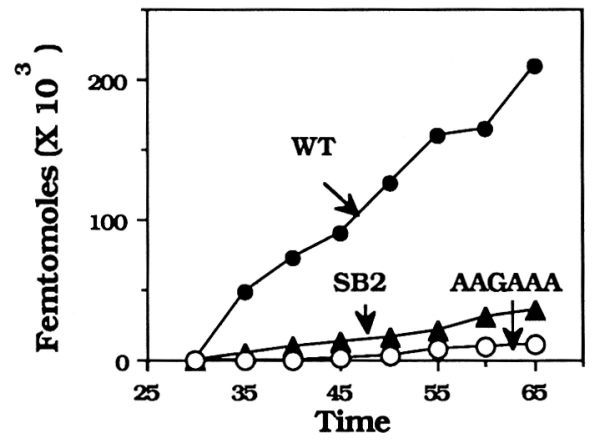

Figure 1. The presence of a poly(A) site is required for maximal in vitro splicing of an upstream intron. $(A)$ Structure of RNA substrates. The chimeric substrates MXSVL WT and MXSVL AAGAAA contain an intron derived from the adenovirus major late transcription unit described previously as MINX (Zillmann et al. 1988), here designated as MX (solid region), followed by the poly(A) site from the SV40 late transcription unit, here designated as SVL (hatched region). MXSVL AAGAAA contains a single point mutation in the hexanucleotide consensus sequence required for polyadenylation. SB2 RNA contains the same intron as MXSVL and a second complete exon of approximately the same length as that in MXSVL (Robberson et al. 1990). (B) Time course of processing reactions. RNAs were incubated under low magnesium processing conditions (estimated final concentration of $<0.25 \mathrm{~mm}$ magnesium for the indicated times. Equal amounts of each precursor were utilized. RNA products were displayed on a $5 \%$ denaturing polyacrylamide gel. Polyadenylated but not spliced $(A+)$, spliced but not polyadenylated $(S+)$, and spliced and polyadenylated $(A+S+)$ RNAs are indicated for each reaction. The sizes of the RNA substrates and products are as follows: (MXSVL WT and AAGAAA precursor RNA), 359; (A +) 304; $(\mathrm{S}+$ ) 239; $(\mathrm{A}+\mathrm{S}+$ ) 184; (SB2 precursor RNA) 414; ( + ) 294. (C) Quantitation of splicing activity. The gel in $B$ was scanned in a Betagen Betascope 603 blot analyzer, and radioactivity in each band was converted to femtomoles product and plotted versus reaction time. For SB2 and MXSVL AAGAAA RNAs, total splicing was calculated as the amount of $\mathrm{S}+\mathrm{RNA}(\boldsymbol{\Delta}$ and $O$, respectively) and for MXSVL WT RNA, as the sum of $\mathrm{S}+$ and $\mathrm{S}+\mathrm{A}+$ RNA species (O).

served under these conditions (Fig. 3A), and little wildtype precursor RNA processed via initial polyadenylation. Both wild-type and mutant precursor RNA were spliced. Quantitation of wild-type and mutant splicing indicated that both were spliced with equal efficiency and kinetics (Fig. 3B). Thus, high magnesium uncoupled 
A
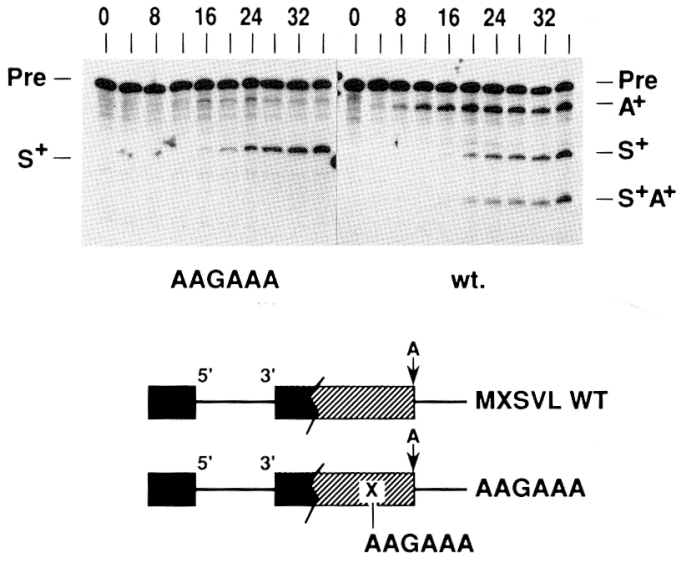

B

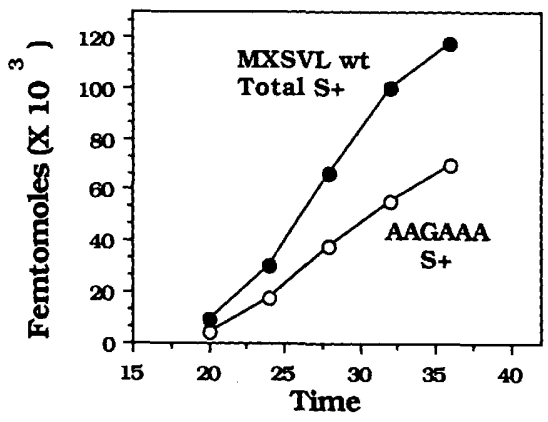

C

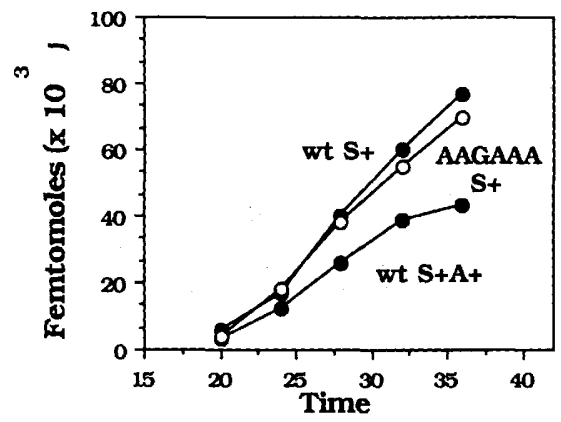

Figure 2. Splicing of chimeric RNAs at moderate magnesium concentration also indicates a relationship between splicing and polyadenylation. $(A)$ MXSVL WT and MXSVL AAGAAA were incubated for the indicated times at a final concentration of $1.5 \mathrm{~mm}$ magnesium. Product species and sizes are as in Fig. 1. (B) Quantitation of splicing activity. Total splicing was calculated as the amount of $\mathrm{S}+$ RNAs for MXSVL AAGAAA $(O)$ and the sum of $\mathrm{S}+$ and S+A RNAs for MXSVL WT (O). (C) Comparison of the splicing of each spliced product RNA. The amount of spliced mutant RNA (AAGAAA $S+$, O) was compared with the amount of sliced but not polyadenylated RNA $(S+, \mid)$ and spliced and polyadenylated RNA $(\mathrm{S}+\mathrm{A}+, \mathbf{0}$, bottom curvel produced during reaction of wild-type RNA. the effect of a poly(A) site on removal of an upstream intron. We suggest that maximal stimulation of splicing by a poly(A) site occurs only under conditions in which polyadenylation is efficient and precedes splicing.

\section{Splicing of only the last intron is dependent} on polyadenylation

The relationship between polyadenylation and splicing was also tested with a chimeric RNA containing two introns (Fig. 4). To avoid potential complications due to different strengths of splicing signals from different introns, the utilized two-intron substrates contained a duplicated MINX splicing unit, such that both $5^{\prime}$ - and 3'splice sites were identical (Fig. 4A). To facilitate identification of the processed product RNAs, the size of the two introns was modified by internal linker addition. We refer to these precursors as MX2SVL WT and MX2SVL AAGAAA. Similar to the single-intron chimeric RNA, MX2SVL WT RNA was able to undergo both splicing and polyadenylation within the same RNA molecule (Fig. 4B). A variety of products were evident from the processing of wild- type RNA. Prominent among these was polyadenylated RNA (denoted $\mathrm{A}+$ ) appearing as early as 10 min and later disappearing, suggesting conversion to other species. Spliced RNA resulting from the removal of intron one appeared next (15 min). This RNA was observed as both poly $(\mathrm{A})^{+}$RNA (denoted $\mathrm{S} 1+\mathrm{A}+$ in Fig. 4B) and as poly(A) ${ }^{-}$RNA (denoted S1 +), indicating that intron 1 removal occurred without prior polyadenylation. Intron 2 removal was observed by $25 \mathrm{~min}$. The majority of this RNA species was polyadenylated, suggesting that intron 2 removal, but not intron 1 removal, occurred after polyadenylation. Intron 2 removal also followed intron 1 removal despite the fact that both introns contained the same sequences. Thus, removal of intron 2 was constrained in these two intron precursor RNAs.

Mutant two-intron RNA was not polyadenylated. Two major RNAs representing splicing to remove intron 1 (S1 + RNA) and splicing to remove both introns $(\mathrm{S} 1+\mathrm{S} 2+\mathrm{RNA})$ were observed. The latter appeared considerably after the former. The amount of splicing of intron 2 in both wild-type and mutant RNA is compared in Figure 4C. Quantitation indicated that intron 2 was removed from wild-type RNA more efficiently than from mutant RNA. The rate of splicing of the second intron in wild- type RNA was estimated to be three times the rate of mutant RNA. Thus, the presence of a poly|A) site resulted in greater splicing of the upstream intron in precursor RNAs with either single or multiple introns. In 

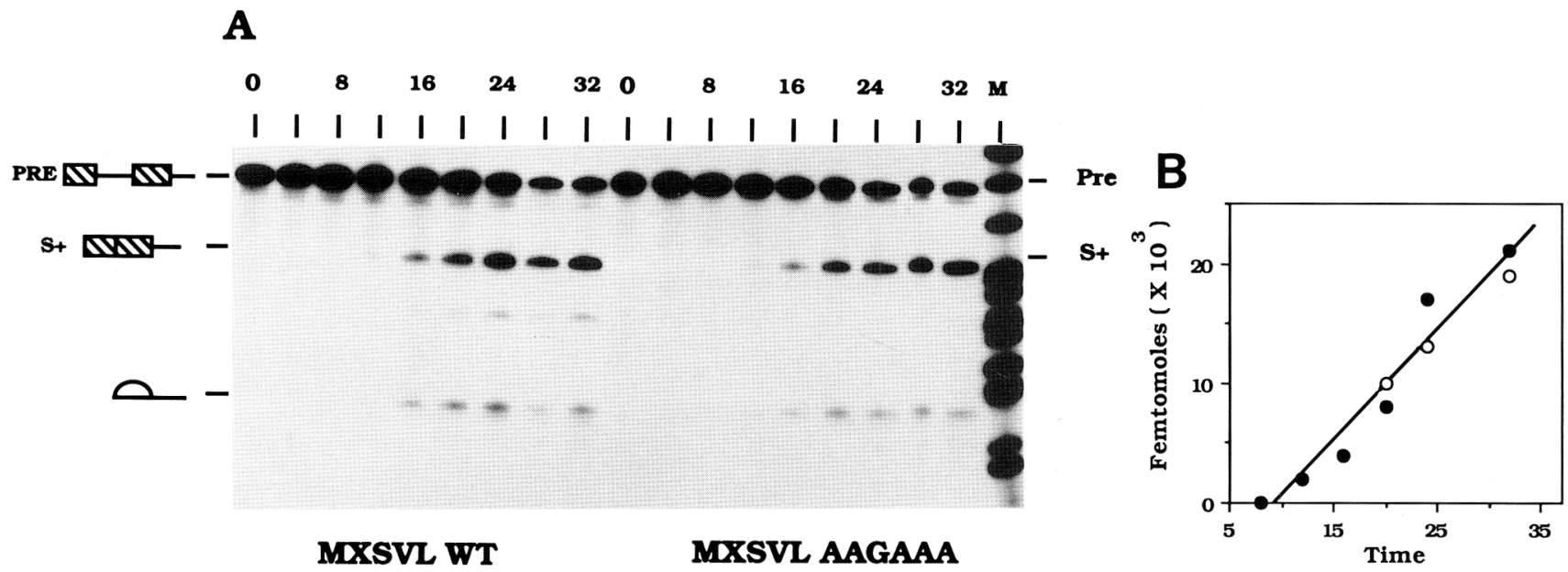

Figure 3. Splicing activity of chimeric RNA at high magnesium concentration is uncoupled from polyadenylation. $(A)$ MXSVL WT and MXSVL AAGAAA were incubated for the indicated times at a final concentration of $4 \mathrm{~mm}$ magnesium. Species are marked as in Fig. 1. (B) Quantitation of splicing activity. The amount of spliced RNA from wild-type RNA (O) is compared with that of mutant RNA 101.

addition, removal of intron 2 was detected considerably earlier for wild-type than for mutant RNA.

The above analysis indicated that removal of intron 2 in a two-intron precursor RNA occurred after both polyadenylation and removal of intron 1 , despite the fact that the splicing signals in introns 1 and 2 were identical. These results suggest that removal of intron 2 was being temporally regulated. To investigate further the splicing of precursor RNAs with multiple introns, we constructed three intron substrates that contained a triplicated MINX splicing unit and a terminal poly(A) site. All of the $5^{\prime}$ - and $3^{\prime}$-splice sites within this construct, MX3SVL, are identical (Fig. 5A).

As with the preceding substrates, MX3SVL WT readily polyadenylated and spliced (Fig. 5B|, producing a complicated array of product species. Individual bands were excised from the gel and analyzed by reverse transcription and polymerase chain reaction (PCR) amplification. Identification of each species was accomplished by restriction mapping each amplification product (see Materials and methods). Wild-type RNA was spliced to remove all three introns. The kinetics of splicing were quantitated for each of the three introns individually. The identical introns were removed in an ordered fashion, in which removal of intron 1 occurred first, followed, in order, by intron 2 and intron 3 (Fig. 5C). Splicing of the cap-proximal intron appeared at $10 \mathrm{~min}$. Splicing of the internal intron appeared at $20 \mathrm{~min}$ while removal of the final intron appeared at $30 \mathrm{~min}$. This same order of intron removal was observed for mutant MX3SVL containing the AAGAAA mutation. Thus, ordered $5^{\prime} \rightarrow 3^{\prime}$ intron removal was not dependent on a wild-type poly(A) site.

The amount of splicing of introns 1-3 was compared between wild-type and mutant RNA (Fig. 5D). Mutation caused a depression in the amount of the splicing of intron 3. The extent of the depression was comparable to that observed with the single and double-intron constructs in Figures 1-4. In contrast, mutation of the poly|A) site had no discernible effect on the extent or kinetics of removal of intron 1 or 2 . Thus, mutation of the polyadenylation consensus sequence specifically depressed removal of the terminal intron but had no effect on introns farther upstream. This result suggests that regions of precursor RNA are recognized segmentally and that a single unit consists of a neighboring exon/ intron pair.

\section{Discussion}

The processing of most vertebrate pre-mRNAs requires both splicing and polyadenylation. Here, we report results consistent with the idea that splicing and polyadenylation processing factors interact. We observed that mutation of the AAUAAA polyadenylation signal resulted in decreased in vitro splicing of a single-intron precursor RNA capable of both splicing and polyadenylation. We have reported previously that mutation of the $3^{\prime}$-splice site terminating an intron depresses in vitro polyadenylation of a downstream poly(A) site (Niwa and Berget 1990). Thus, mutation of splicing signals depresses polyadenylation, and mutation of polyadenylation signals depresses splicing.

The relationship between splicing and polyadenylation during mRNA biogenesis has been historically unclear. Although a number of polyadenylation mutants have been isolated and characterized, most of these mutants were analyzed only with respect to the effect of the mutation on cytoplasmic RNA levels. To our knowledge, there is no report documenting the effect of such mutations on the splicing of upstream introns. This in vitro study suggests that such analyses will indicate a block to splicing of the last intron in the nucleus. The effect of mutation of the poly(A) site was also observed 
A

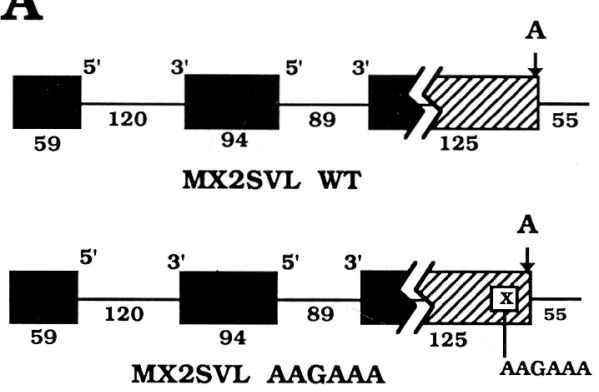

B
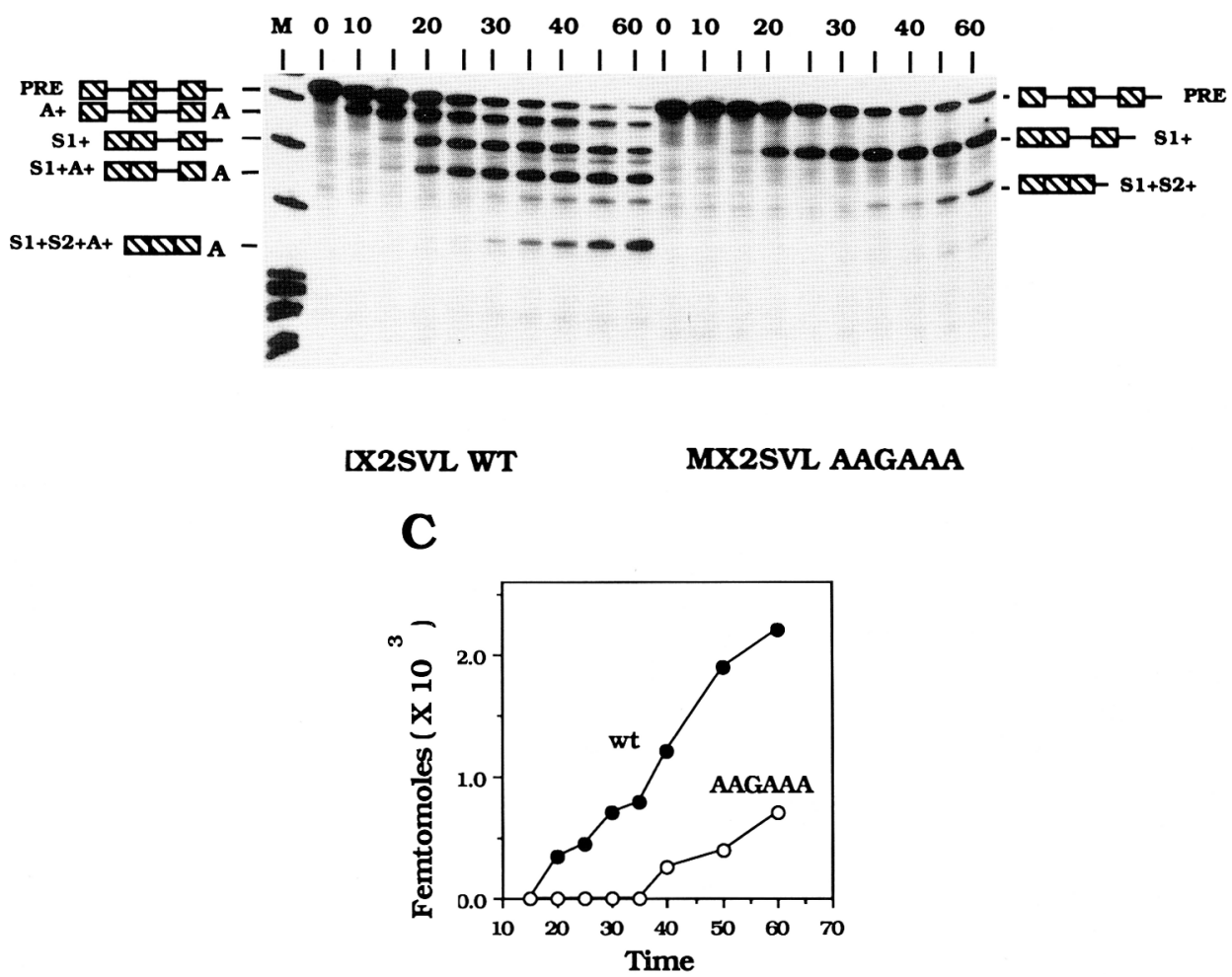

Figure 4. Mutation of the poly(A) consensus sequence depresses removal of the last intron of a two-intron precursor RNA. (A) Chimeric RNAs containing three exons were constructed by duplication of the MX unit depicted in Fig. 1. Only the size of the two introns differed. $(B)$ Time course of reaction. Substrates were incubated under standard conditions (low magnesium) for the indicated times. Reaction products are indicated as follows: $(\mathrm{A}+)$ Polyadenylated but not spliced $\mathrm{RNA}_{;}(\mathrm{S} 1+)$ first intron removed, not polyadenylated; $(S 1+A+)$, first intron removed, polyadenylated; $(S 1+S 2+)$ introns one and two removed, not polyadenylated; $(\mathrm{S} 1+\mathrm{S} 2+\mathrm{A}+)$ introns one and two removed, polyadenylated. Identity of the reaction products was confirmed by restriction digestion analysis of isolated bands previously subjected to reverse transcription and PCR amplification. The sizes of the RNA substrates and products are as follows: (MXSVL WT and AAGAAA precursor RNA) 542; $(\mathrm{A}+)$ 487; (S1 +) 422; (S1 + A +) 367; (S1 + S2 + A +) 278; $(\mathrm{S} 1+\mathrm{S} 2+)$ 333. The unmarked band between the $\mathrm{S} 1+$ and $\mathrm{S} 1+\mathrm{A}+$ bands in the left panel is $\mathrm{S} 2+\mathrm{A}+\mathrm{RNA}, 398$. (C) Quantitation of the removal of the second intron. The total amount of removal of intron 2 from wild-type RNA (O) is compared with that of mutant RNA (O).

with precursor RNAs containing multiple introns. In this case, splicing of only the terminal intron was affected by mutation of the poly(A) site; other introns were spliced normally. Thus, we predict that any resulting block to nuclear splicing that might occur in poly(A) site mutants would apply to only the 3 '-terminal intron. It is possible, however, that experiments of this type will be uninformative because of activation of cryptic downstream poly(A) sites when the normal site is missing or mutated.

Depression of splicing of only the terminal intron supports a modular perspective of precursor RNA recognition. Results analogous to those reported here for $3^{\prime}$-terminal introns were observed for $5^{\prime}$-terminal introns 


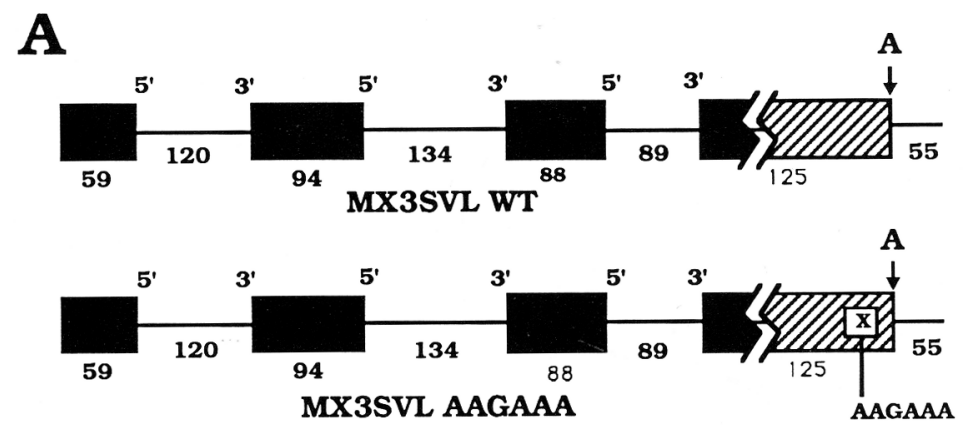

B

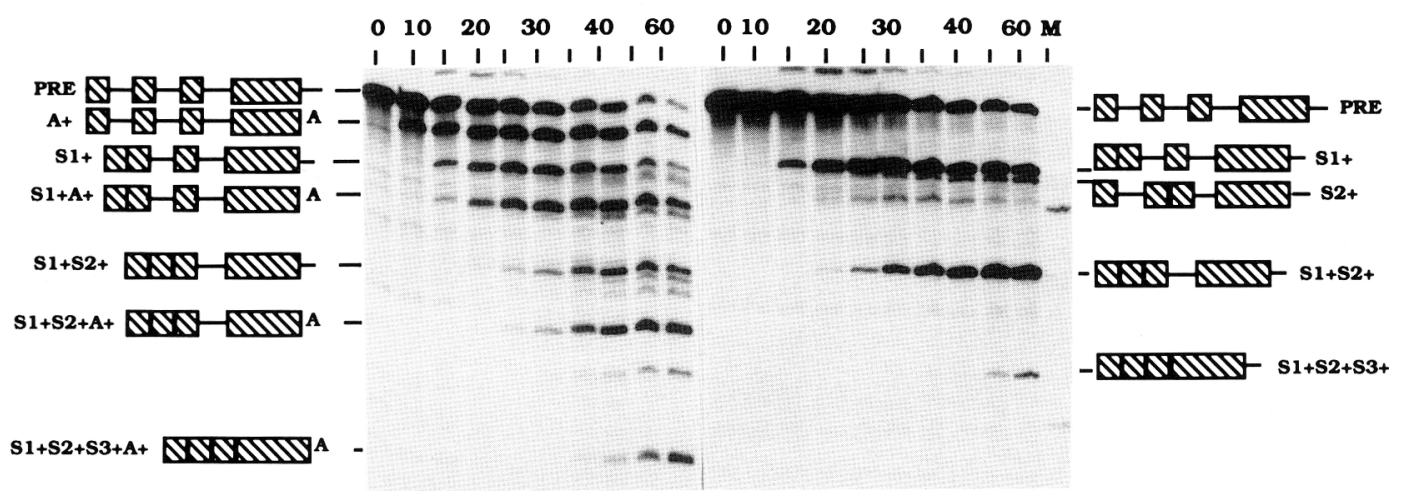

MX3SVL WT

C

Figure 5. Mutation of the poly(A) consensus sequence depressed removal of only the last intron of a three-intron precursor RNA. $|A|$ Structure of substrate RNAs. The chimeric RNAs containing four exons (MX3SVL) were constructed by triplication of the MX unit depicted in Fig. 1. (B) Time course of the reaction. The sizes of the RNA substrates and products are as follows: (MX3SVL WT and AAGAAA precursor RNAs) $764 ;(\mathrm{A}+1$ 709; $(\mathrm{S} 1+) 644 ; \mid \mathrm{S} 2+) 630 ;(\mathrm{S} 1+\mathrm{A}+\mid 589 ;$ $(\mathrm{S} 1+\mathrm{S} 2+) \quad 510 ; \quad(\mathrm{S} 1+\mathrm{S} 2+\mathrm{A}+) \quad 455 ;$

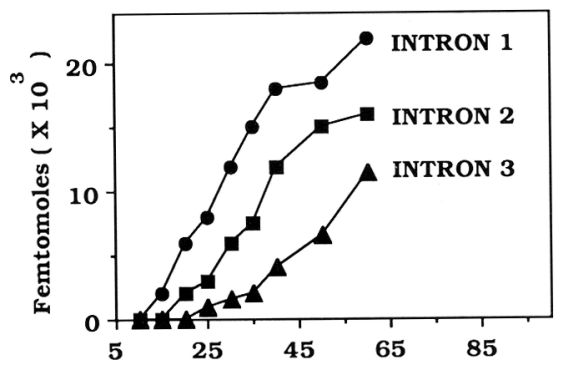

D

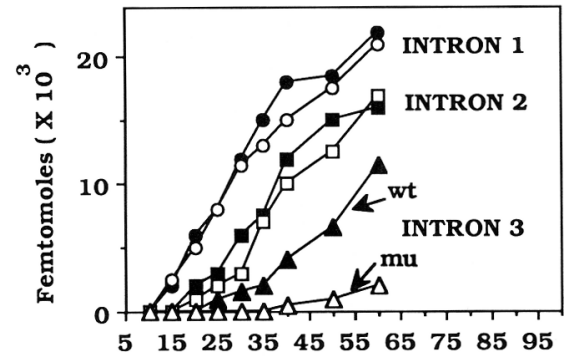

$(\mathrm{S} 1+\mathrm{S} 2+\mathrm{S} 3+) 421 ;(\mathrm{S} 1+\mathrm{S} 2+\mathrm{S} 3+\mathrm{A}+\mid 366$. (C) Order of removal of introns from MX3SVL WT. Quantitation of the splicing as a result of the removal of individual introns was calculated. For each intron, the total splicing resulting from removal of intron 1 (S1+, intron $2(\mathrm{~S} 2+, \mathbf{D})$ and intron $3(\mathrm{~S} 3+, \boldsymbol{\Delta})$ is displayed. $(D)$ Removal of introns 1,2 , and 3 in wild-type $(\boldsymbol{O}, \mathbf{\square}$, and $\boldsymbol{\Delta}$, respectively) and mutant $(O, \square$, and $\triangle$, respectively) RNA.

when the effect of cap on splicing was examined (Ohno et al. 1987). Substitution of the analog ApppG for the normal GpppG cap reduced splicing of the first, but not the second, intron in an in vitro assay with a precursor RNA containing duplicated introns. Again, this result suggests that precursor RNAs are recognized segmentally.

An early in vivo study investigating the relationship between splicing and polyadenylation reported that addition of cordycepin ( $3^{\prime}$-deoxyadenosine) to cells inhib- ited polyadenylation but permitted continued splicing, suggesting that the two processes were uncoupled (Zeevi et al. 1981). Although cordycepin limits the poly(A)addition step of polyadenylation to the addition of a single A residue, it does not prevent the cleavage reaction of polyadenylation. Coupled with the results presented here, this observation suggests that the polyadenylation process required for optimal splicing is not poly(A)-addition but, rather, some earlier event.

We do not know how factors involved in splicing and 
polyadenylation might interact to facilitate recognition of 3 -terminal exons. We reported previously that although a wild-type $3^{\prime}$-splice site was required for maximal polyadenylation of a downstream site, a $5^{\prime}$-splice site was not required. We suggest that whatever interaction occurs, it is between factors that recognize $3^{\prime}$-splice sites and poly|A|sites. It is unclear whether the poly(A) sites that terminate genes with introns are identical to sites terminating genes without introns or if such sites are processed by the same factors. Similarly, it is unclear whether the 3 '- splice signals or the factors that recognize them are different for terminal versus internal introns. The intron in the precursors used here was not a 3 '-terminal intron. We have observed similar results, however, using precursor RNAs containing normal last introns and exons. Attempts to identify factors unique to terminal exons by using UV cross-linking approaches only identified the standard set of polypeptides known to associate with isolated splicing or poly(A) signals (Stolow and Berget 1990). Thus, we do not know whether the interaction that we have observed requires factors other than those characterized by using standard splicing or polyadenylation precursor RNAs. We do suspect that the interaction between splicing and polyadenylation factors implied by our data occurs at the earliest stages of spliceosome assembly. Analysis of single-intron precursor RNAs terminated by a poly(A) site on neutral gels used to resolve splicing complexes revealed the presence of a complex migrating slower than either splicing or polyadenylation complexes after $1 \mathrm{~min}$ of incubation (Niwa and Berget 1991). The presence of this complex suggests the simultaneous presence of both splicing and poly(A) factors at a very early stage of substrate recognition.

Our chimeric constructs contain strong splicing and polyadenylation signals. In this case, splicing and polyadenylation factors might interact directly. When either of the processing signals is weak, an additional factor(s) might be required. For example, doublesex $(d s x)$ mRNA in Drosophila contains a differentially utilized terminal exon characterized by weak splicing signals (Hedley and Maniatis 1991; Hoshijima et al. 1991; Mattox and Baker 1991). Recognition of this exon requires the transformer and transformer-2 gene products that bind to sequences within the exon. It has been suggested that transformer and transformer- 2 protein function by promoting interaction between splicing and polyadenylation factors.

We have reported previously that simple single-intron precursor RNAs are processed via two pathways in vitro (Niwa and Berget 1991). In one pathway, polyadenylation occurs first and leads to the production of doubly processed RNA. In the second pathway, splicing occurs first. RNA processed by this pathway is recalcitrant to polyadenylation, suggesting that it is a dead-end pathway. The relative amount of precursor RNA processed via the two pathways is dependent on the magnesium concentration employed in the assay: Lower magnesium favors the branch that polyadenylated first and produced product RNA that had undergone both reactions. In this report we noticed that the effect of a poly(A) site mutation on removal of the upstream intron could be modulated by magnesium. At the low magnesium concentrations that favored polyadenylation, mutation depressed splicing at least 10-fold. At higher magnesium, less inhibition was observed. At $4 \mathrm{~mm}$ magnesium, conditions that completely inhibit polyadenylation, the poly(A) site mutation had essentially no effect on splicing. Thus, interaction between splicing and polyadenylation required not only wild-type poly(A) signals but also assay conditions under which poly(A) signals were maximally recognized by splicing/polyadenylation factors.

We have suggested previously that the exon is the unit of recognition during assembly of the vertebrate spliceosome (Robberson et al. 1990). For internal exons, we suggested that splicing factors concertedly recognize splicing signals at both the $3^{\prime}$ and $5^{\prime}$ ends of the exon. Such a view of spliceosome assembly requires special mechanisms to recognize $3^{\prime}$-terminal exons, which begin with a 3 -splice site and terminate with a poly(A) site, and for $5^{\prime}$-terminal exons, which begin with a cap and end with a $5^{\prime}$-splice site. The data reported here and that mentioned above by Ohno et al. (1987) indicate that removal of terminal introns is affected by signals residing at the other end of the terminal exon; the cap for first introns and the poly(A) site for last introns. These observations are consistent with the exon being the recognition unit. From this perspective, a multi-exon precursor RNA contains three types of exons: a $5^{\prime}$ exon demarcated by a cap and a $5^{\prime}$-splice site; internal exons demarcated by a $3^{\prime}$ splice site and a $5^{\prime}$-splice site; and a $3^{\prime}$ exon demarcated by a $3^{\prime}$-splice site and a poly(A) site.

Most vertebrate pre-mRNA contains multiple introns. Here, we observed that when multiple identical introns were present, in vitro splicing of specific introns occurred in an orderly manner. Splicing was processive in the $5^{\prime} \rightarrow 3^{\prime}$ direction beginning quickly with the capproximal intron. One other study with duplicated introns also reported a $5^{\prime} \rightarrow 3^{\prime}$ sequential intron removal in vitro (Ohno et al. 1987). A third study saw equal rates of removal (Christofori et al. 1987). In this case, however, both introns were removed quite slowly; and intron 1 removal may have been impaired because 32 of the 40 nucleotides comprising exon 1 were derived from vector polylinker. Sequential removal is supported by in vivo data showing that splicing of introns distal to the poly|A| site occurs during transcription (Beyer and Ohsheim 1988). It contradicts data from steady-state analysis of nuclear intermediates of cellular genes that indicate a nonprocessive pattern of intron removal (Ryffel et al. 1980; Tsai et al. 1980; LeMaire and Thummel 1990.). No analysis of the in vivo splicing pattern of an engineered gene with multiple identical introns has been reported. It is possible that when effects of splice site strength are neutralized by duplication, a processive pattern of intron removal will be observed in vivo. As discussed above, analysis of the splicing properties of mutant RNAs altered in their $5^{\prime}$ - or $3^{\prime}$-terminal exons suggests that precursor RNAs are recognized segmentally. Such segmental recognition would be consistent with a processive removal of introns. Several years ago, Aebi et al. (1986) 
suggested a first-come-first-serve model for splice site recognition in which splice sites are chosen within segments of an RNA as it is revealed by transcription. The segmental nature of precursor recognition implied by our data supports a first-come-first-serve view of splice site recognition with the suggested modification that the recognized segment is the exon, rather than the intron.

\section{Materials and methods}

Precursor RNAs

All MXSVL chimeric precursor RNAs were derived from the MINX family of adenovirus precursor RNAs described previously by this laboratory (Zillmann et al. 1988). Each contained an identical 220-nucleotide splicing cassette including a 59-nucleotide first exon, a 120-nucleotide intron, and a 38-nucleotide truncated second exon. Sequences from the SV40 late transcription unit including the poly(A) site were fused within exon 2 to produce the basic one-intron MXSVL precursor RNA. The complete sequence of MXSVL is given in Niwa et al. (1990). A mutant MXSVL containing a point mutation was constructed with a mutant SV40 late cassette provided by $\mathrm{M}$. Wickens /University of Wisconsin|. The wild-type and mutant RNAs were slightly smaller than the MXSVL described in Niwa et al. (1990) because the SV40 information included only nucleotides -58 to +55 of the poly(A)-addition site.

\section{Splicing reactions}

Splicing reactions with MXSVL precursor RNAs were carried out under conditions that represent a compromise between the maximal standard conditions for splicing and polyadenylation as described previously (Niwa et al. 1990). The magnesium in the reaction varied from 0.25 to $4.0 \mathrm{~mm}$. Unless indicated otherwise, the standard reaction contained $0.5 \mathrm{~mm}$ magnesium. Each reaction contained $3^{\prime}$ dATP to aid in visualization of products that had undergone polyadenylation cleavage. In this paper we refer to RNA molecules that have undergone cleavage and addition of a single $A$ residue as having been polyadenylated. Products of reaction of MXSVL have been characterized previously (Niwa et al. 1990). Reaction products from three- and four-exon constructs were excised as single bands from polyacrylamide gels and reverse-transcribed by using a primer specific to the SV40 poly(A) site. Reverse transcripts were amplified by PCR with oligonucleotides specific to the first and last ex ons. Each amplification product was identified by restriction enzyme analysis. Each intron in the utilized precursors contained a unique restriction endonuclease site.

\section{Quantitation of splicing reactions}

Polyacrylamide gels of RNA products from processing reactions were quantitated by scanning in a Betagen Betascope 603 blot analyzer. Amount of product was calculated from a knowledge of the specific activity of the $\left[{ }^{32} \mathrm{P}-\right] \mathrm{UTP}$ labeled precursor RNA, $\mathrm{U}$ content of each RNA species, and the determined efficiency of counting in the instrument $(15 \%)$. When two substrates were compared, substrates were prepared carefully to the same specific activity and reacted with the same reaction/extract mix. At least five determinations were made for each comparison to ensure reproducibility. The plots shown are from the quantitation of the displayed gel. Polyadenylation and splicing rates were identical over at least a 20 -fold concentration of precursor RNA, indicating that reactions were performed under nonsat- urating conditions. Precursor RNAs both react and are degraded in processing extracts. Analysis of total counts for each substrate during reaction indicated that each of the precursors used in this study had a similar half-life in extract. Rates were calculated from early time points in the reaction before product decay contributed significantly to disappearance of total counts.

The publication costs of this article were defrayed in part by payment of page charges. This article must therefore be hereby marked "advertisement" in accordance with 18 USC section 1734 solely to indicate this fact.

\section{Acknowledgment}

This work was supported by the American Cancer Society.

\section{References}

Aebi, M., H. Hornig, R.A. Padgett, J. Reiser, and C. Weissmann. 1986. Sequence requirements for splicing of higher eucaryotic nuclear pre-mRNAs. Cell 47: 555-565.

Berget, S.M. and P.A. Sharp. 1978. Structure of late adenovirus 2 heterogeneous nuclear RNA. J. Mol. Biol. 129: 547-559.

Beyer, A. and Y.N. Ohsheim. 1988. Splice site selection, rate of splicing, and alternative splicing on nascent transcripts. Genes \& Dev. 2: 754-765.

Christofori, G. and W. Keller. 1988. 3' cleavage and polyadenylation of mRNA precursors in vitro requires a poly $(\mathrm{A})$ polymerase, a cleavage factor, and a snRNP. Cell 54: 875-889.

Christofori, G., D. Frendewey, and W. Keller. 1987. Two spliceosomes can form simultaneously and independently on synthetic double-intron messenger RNA precursors. $E M B O J$. 6: $1747-1755$.

Ford, J.P. and M.-T. Hsu. 1978. Transcription pattern of in vivolabeled late simian virus 40 RNA: Equimolar transcription beyond the mRNA 3 ' terminus. I. Virol. 28: 795-801.

Gilmartin, G.M., M.A. McDevitt, and J.R. Nevins. 1988. Multiple factors are required for specific RNA cleavage at a poly(A) addition site. Genes \& Dev. 2: 578-587.

Green, M.R., T. Maniatis, and D.A. Melton. 1983. Human betaglobin pre-mRNA synthesized in vitro is accurately spliced in Xenopus oocyte nuclei. Cell 32: 681-694.

Hedley, M.L. and T. Maniatis. 1991. Sex-specific splicing and polyadenylation of dsx pre-mRNA requires a sequence that binds specifically to tra-2 protein in vitro. Cell 65: $579-586$.

Hoshijima, K., K. Inoue, I. Higuchi, H. Sakamoto, and Y. Shimura. 1991. Control of doublesex alternative splicing by transformer and transformer-2 in Drosophila. Science 252: 833-836.

Huang, M.T.F. and C.M. Gorman. 1990. Intervening sequences increase efficiency of RNA $3^{\prime}$ processing and accumulation of cytoplasmic RNA. Nucleic Acids Res. 18: 937-947.

Koehavong, P., R. Gattoni, J.M. LeMoullec, M. Jacob, and J. Stevenin. 1982. The orderly splicing of the adenovinus- 2 major late transcript. Nucleic Acids Res. 10: 1215-1229.

Kramer, A. 1988. Presplicing complex formation requires two proteins and U2 snRNP. Genes \& Dev. 2: 1155-1167.

LeMaire, M.F. and C.S. Thummel. 1990. Splicing precedes polyadenylation during Drosophila E74A transcription. Mol. Cell. Biol. 710: 6059-6063.

Mariman, E.C.M., R.J. van Beek-Reinders, and W.J. van Venrooij. 1983. Alternative splicing pathways exist in the formation of adenoviral last messenger RNAs. J. Mol. Biol. 169: 239-256.

Mattox, W. and B.S. Baker. 1991. Autoregulation of the splicing of transcripts from the transformer-2 gene of Drosophila. 
Genes \& Dev. 5: 786-796.

Nevins, J.R. and J.E. Darnell, Jr. 1978. Steps in the processing of Ad2 mRNA: Poly $\mid \mathrm{A})^{+}$sequences are conserved and poly $(\mathrm{A})$ addition precedes splicing. Cell 15: 1477-1493.

Niwa, M. and Berget, S.M. 1991. Polyadenylation precedes splicing in vitro. Gene Expression 1: 5-14.

Niwa, M., S.D. Rose, and S.M. Berget. 1990. In vitro polyadenylation is stimulated by an upstream intron. Genes \& Dev. 9: 1552-1559.

Ohno, M., H. Sakamoto, and Y. Shimura. 1987. Preferential excision of the $5^{\prime}$ proximal intron from mRNA precursors with two introns as mediated by the cap structure. Proc. Natl. Acad. Sci. 84: 5187-5191.

Robberson, B.L., G. Cote, and S.M. Berget. 1990. Exon definition may participate in splice site recognition in multi-exon RNAs. Mol. Cell. Biol. 10: 84-94.

Ryffel, G.U., T. Wyler, D.B. Muellener, and R. Weber. 1980. Identification, organization, and processing intermediates of the putative precursors of Xenopus vitellogenin messenger RNA. Cell 19: 53-61.

Sheets, M.D., S.C. Ogg, and M.P. Wickens. 1990. Point mutations in AAUAAA and the poly $(\mathrm{A})$ addition site: Effects on the accuracy and efficiency of cleavage and polyadenylation in vitro. Nucleic Acids Res. 18: 5799-5805.

Stolow, D.T. and S.M. Berget. 1990. UV cross-linking of polypeptides associated with 3 '-terminal exons. Mol. Cell. Biol. 10: $5937-5944$.

Takagaki, Y., L.C. Ryner, and J.L. Manley. 1988. Separation and characterization of a poly(A) polymerase and a cleavage/ specificity factor required for pre-mRNA polyadenylation. Cell 52: 731-742.

Tsai, M.-J., A.C. Ting, J.L. Nordstrom, W. Zimmer, and B.W. O'Malley. 1980. Processing of high molecular weight ovalbumin and ovomucoid precursor RNAs to messenger RNA. Cell 22: 219-230.

Villereal, L.P. and R.T. White. 1983. A splice junction deletion deficient in the transport of RNA does not polyadenylate nuclear RNA. Mol. Cell. Biol. 3: 1381-1388.

Weber, J., J.-M. Blanchard, H. Ginsberg, and J.E. Darnell Jr. 1980. Order of polyadenylic acid addition and splicing events in early adenovirus mRNA formation. J. Virol. 33: 286-291.

Zeevi, M., J.R. Nevins, and J.E. Darnell Jr. 1981. Nuclear RNA is spliced in the absence of poly $(A)^{-}$addition. Cell 26: 39-46.

Zillmann, M., M. Zapp, and S.M. Berget. 1988. Gel electrophoretic isolation of splicing complexes containing U1 small nuclear ribonucleoprotein particles. Mol. Cell. Biol. 8: 814 821. 


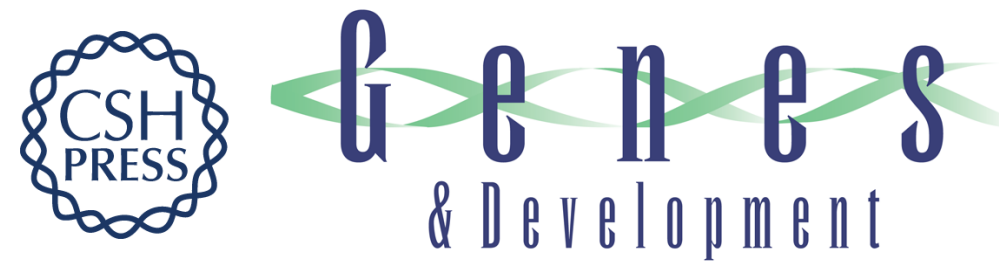

\section{Mutation of the AAUAAA polyadenylation signal depresses in vitro splicing of proximal but not distal introns.}

M Niwa and S M Berget

Genes Dev. 1991, 5:

Access the most recent version at doi:10.1101/gad.5.11.2086

References This article cites 30 articles, 12 of which can be accessed free at:

http://genesdev.cshlp.org/content/5/11/2086.full.html\#ref-list-1

License

Email Alerting

Service

Receive free email alerts when new articles cite this article - sign up in the box at the top right corner of the article or click here.

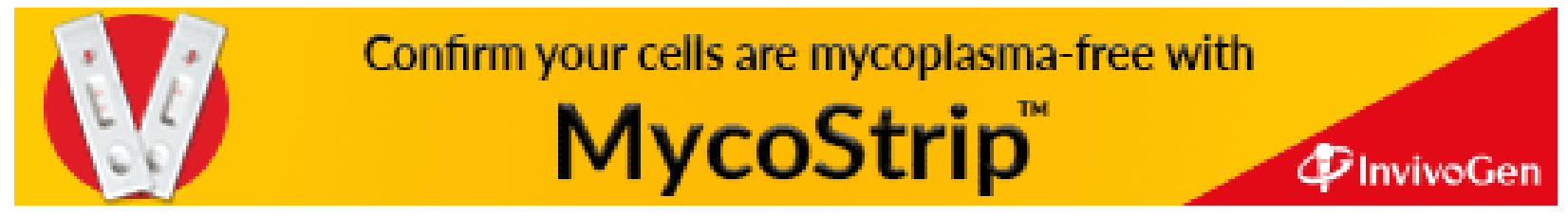

